\title{
A multi-color based features from facial images for automatic ethnicity identification model
}

\author{
Mohd Zamri Osman ${ }^{1}$, Mohd Aizaini Maarof ${ }^{2}$, Mohd Foad Rohani ${ }^{3}$, Nilam Nur Amir Sjarif ${ }^{4}$, \\ Nor Saradatul Akmar Zulkifli ${ }^{5}$ \\ ${ }^{1,5}$ Soft Computing Research \& Intelligent System (SPINT), Faculty of Computing, \\ Universiti Malaysia Pahang (UMP), Malaysia) \\ ${ }^{2,3}$ Information Assurance and Security Research Group (IASRG), School of Computing, \\ Universiti Teknologi Malaysia (UTM), Malaysia \\ ${ }^{4}$ Advanced Informatics School, Level 5, Menara Razak, Universiti Teknologi Malaysia (UTM), Malaysia
}

\begin{tabular}{l} 
Article Info \\
\hline Article history: \\
Received Jul 28, 2019 \\
Revised Oct 29, 2019 \\
Accepted Nov 13, 2019 \\
\hline
\end{tabular}

\section{Keywords:}

Demographic image analysis Ethncity identification

Skin color spaces

Skin detection

Soft-biometric analysis

\begin{abstract}
Ethnicity identification for demographic information has been studied for soft biometric analysis, and it is essential for human identification and verification. Ethnicity identification remains popular and receives attention in a recent year especially in automatic demographic information. Unfortunately, ethnicity identification technique using color-based feature mostly failed to determine the ethnicity classes accurately due to low properties of features in color-based. Thus, this paper purposely analyses the accuracy of the color-based ethnicity identification model from various color spaces. The proposed model involved several phases such as skin color feature extraction, feature selection, and classification. In the feature extraction process, a dynamic skin color detection is adapted to extract the skin color information from the face candidate. The multi-color feature was formed from the descriptive statistical model. Feature selection technique applied to reduce the feature space dimensionality. Finally, the proposed ethnicity identification was tested using several classification algorithms. From the experimental result, we achieved a better result in multi-color feature compared to individual color space model under Random Forest algorithm.
\end{abstract}

Copyright $@ 2020$ Institute of Advanced Engineering and Science. All rights reserved.

\section{Corresponding Author:}

Mohd Zamri Osman,

Soft Computing Research \& Intelligent System (SPINT),

Faculti of Computer Systems \& Software Engineering,

Universiti Malaysia Pahang (UMP), Pahang, Malaysia.

Email: zamriosman@ump.edu.my

\section{INTRODUCTION}

The human face is crucial in identifying the person because it is rich in information about personal characteristics. Previously, information from the human face has gained much attention especially in the soft-biometric analysis [1]. Face providing several important information, including personal identity, gender [2], age [2-5], ethnicity [6, 7], skin tones and emotional expression [8-11]. Ethnicity identification is the key demographic attribute, and it plays an important role in many applications [12-14]. Thus, a human face must be detected and exploited in order to analyze the facial information. Ethnicity identification is challenging because of its ambiguity and complexity. To address the issues, several ethnicity identification models have been introduced to enhance the performance in categorizing different ethnic group. Color or chromatic-based feature has been used in classifying ethnic group due to some reason. It is based on the justification that every person has different skin range, and might include the ethnicity properties, especially in controlled condition. The author [14] stressed that the justification of chromatic is less significant lies on 
the visual appliance of chromatic attributes. Although chromatic-based feature had some improvement in detection rate, the results may still not satisfactory due to minimal feature derived from the skin color. Several researchers utilized color-based such YCoCg-R in watermarking technique [15], HSV in shadow detection [16], cloth retrieval [17] and license plate detection [18].

The ethnic group generally is formed based on the biological unit which made up from skin color, head shape, hair, face shape, and blood type. Ethnic generally is broken into three main categories: European (Caucasoid), African (Negroid), and Asian (Mongoloid) [19-21]. Asian skin is yellowish while the European is light or brown. On the other hand, African skin color is dark. Ethnic skin color varies because of melanin quantity under the skin. Skin color or chromatic-based feature for ethnicity identification has been studied previously by [7, 22-25], and their results appeared acceptable. However, several justifications that different ethnic might share similar skin tones. For example, skin tone from Southern Indian, and African might be clustered in the same dark skin tone even though they are from different ethnics. The worst case is that the skin tone is sensitive to uncontrolled illuminations that can produce errors [26]. Ethnicity identification based on a chromatic feature from the previous articles mentioned in psychological [26], which that skin tone does not have a relationship to ethnic representation. The justification is based on the psychological view with less of evaluation on the various color-based features. Brooks and Gwinn [27] examined the correlation between 'White' and 'Black' people under the skin tone characteristic. They perceived that ethnicity does not determine by the skin tone. Though, the faces were converted into a grayscale format without presenting the result within the various color space model such as RGB, YCbCr, HSV, YUV, CIE Lab and CIE Luv.

This paper investigates the interrelation between ethnicity and color by proposing a new method in finding the optimal color-based feature from various color spaces for improving automatic ethnicity identification model under multi-class ethnic group. The proposed method employs three phases: skin color feature extraction, feature selection, and a classification phase. The skin colors were employed using several color spaces such as RGB, YCbCr, HSV, YUV, CIE Lab and CIE Luv. The rest of this paper is organized as follows: Section II provides a brief introduction to the methodology of the proposed model. Section III describes the results and analysis, and Section IV concludes the paper.

\section{RESEARCH METHOD}

This study aims to analyze the performance of ethnicity identification based on the color feature from the face images. The proposed ethnicity identification model is divided into three major phases. The first phase is skin color feature extraction which exploits the color properties from the face image using skin color detection. The second phase is feature selection were applied to reduce the dimensionality of the obtained features from the first phase. The last phase is the classification which applied to several classification algorithms.

\subsection{Skin Color Feature Extraction}

The skin color was carried out based on the color of each face skin. We used our skin color detection which introduced in [28-30]. The skin color detection was applied as the pre-processing stage to extract the skin color information. Here, viola-jones face detection algorithm was employed to find the human face, extract the skin thresholds and classify the input image. Extraction of color-based from the face-skin was carried out. The face region was then converted into five color spaces which are YCbCr, YIQ, HSV, CIE Luv, and CIE Lab. The Y, Cb, and $\mathrm{Cr}$ are the skin feature vector for $\mathrm{YCbCr}$ color channels obtained from the face-skin region. The skin pixel from the face-skin region extracted in the form of a vector. In the end, these extracted skin color pixels were presented into a new feature vector by calculated using a descriptive statistical feature. There are nine descriptive statistical feature utilized for ethnicity features which are: minimum, maximum, mean, median, mode, standard deviation, variance, skewness, and kurtosis. The detail description with the formula of the descriptive statistics is presented in Table 1. All nine-descriptive statistic feature were calculated based on each skin vector of the color channel. A total of 162 features were generated from 18 color channels $(3 \times 6$ color space including RGB), and nine descriptive statistics.

Where,

$\mathrm{n}$ is the number of skin pixels,

$\mathrm{p}$ is the nth skin pixels

li lower limit of modal class

f0 frequency of the preceding modal class, and

i class interval of modal class 
Table 1. Description Statistics for Color Features

\begin{tabular}{lcc}
\hline No. & Feature & Formula \\
\hline$f 1$ & Minimum & The lowest pixel value \\
$f 2$ & Maximum & $\bar{p}=\frac{\sum p}{n}$ \\
$f 3$ & Mean & $\frac{n+1}{2}$ \\
$f 4$ & Median & $l_{1}+\frac{f_{1-f_{0}}}{2 f_{1}-f_{0}-f_{2}} \times i$ \\
$f 5$ & Mode & $s=\sqrt{\frac{\sum(p-\bar{p})}{n-1}}$ \\
$f 6$ & Standard deviation & $s^{2}=\frac{\sum(p-\bar{p})}{n-1}$ \\
$f 7$ & Variance & $(n-1)(n-2) \sum\left(\frac{p-\bar{p}}{s}\right)^{2}$ \\
$f 8$ & Skewness & $\alpha^{2}=\frac{n(n-1)}{(n-1)(n-1)(n-3)}$ \\
$f 9$ & Kurtosis & $\left.\sum\left(\frac{p-\bar{p}}{s}\right)-\frac{3(n-1)^{2}}{(n-2)(n-3)}\right\}$ \\
& & $\sum$
\end{tabular}

The $f 1$ until $f 9$ indicates the feature vector for each color channels. For instance, $f 1 \_R, f 1 \_G, f 3 \_B$ respectively for $R, G$ and $B$ color channels which will formed nine color features from $R G B$ color space under maximum descriptive statistical feature. The following feature vector $f 2$ will having the same process of vectorization until feature vector of $f 9$. The process of color-based extraction using our previous skin color detection and calculation of descriptive statistical feature is illustrated in

Figure 1. The ethnicity identification model utilized the skin color from the face candidate as the key feature. Extraction of skin color from the face was carried out by converting the skin vector into six different color spaces. At the end of this phase, a feature space obtained where six color spaces were performed to characterize the human face for classifying the ethnic group. Feature space formed 162 attributes consisted of the descriptive statistical feature of each color channels from all six color spaces.

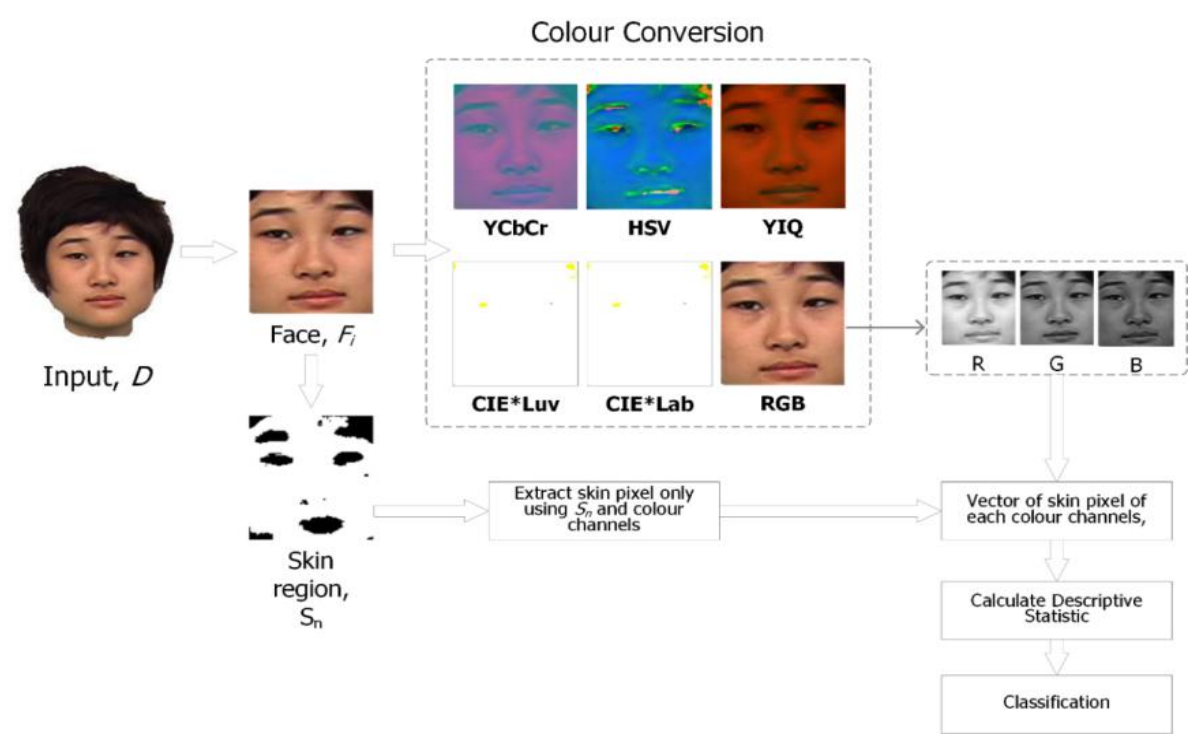

Figure 1. The flowchart of the extraction skin color using skin color detection algorithm, and descriptive statistical features

\subsection{Feature Selection using Wrapper-based Subset Evaluator}

This phase implemented a feature selection method which aims to reduce the dimensionality of feature space. The feature selection chose only the most relevant features by removing irrelevant features by using wrapper subset evaluation and genetic algorithm (GA) search method. Concerning the number of 
images used for this study, the ethnic groups for each ethnic group were specified accordingly for training and testing. A total of 343 face images are for Asian, 929 face images for Caucasian, 216 African-American, and 62 face images for Hispanic. The Accuracy of the Ethnicity Identification Model based on Different Color Spaces (Trainset) as shown in Table 2.

Table 2. The Accuracy of the Ethnicity Identification Model based on Different Color Spaces (Trainset)

\begin{tabular}{cccccccc}
\hline \multirow{2}{*}{ Classifier } & & \multicolumn{7}{c}{ Color Features } \\
& RGB & $\mathrm{YC}_{\mathrm{b}} \mathrm{C}_{\mathrm{r}}$ & YIQ & HSV & CIE Lab & CIE Luv & All \\
\hline Naïve Bayes & 0.683 & 0.690 & 0.715 & 0.627 & 0.711 & 0.739 & 0.686 \\
BayesNet & 0.692 & 0.685 & 0.720 & 0.733 & 0.699 & 0.716 & 0.709 \\
SVM & 0.599 & 0.601 & 0.756 & 0.688 & 0.610 & 0.608 & 0.599 \\
$k$-NN & 0.818 & 0.857 & 0.871 & 0.858 & 0.876 & 0.894 & 0.899 \\
Random Forest & 0.845 & 0.887 & 0.870 & 0.885 & 0.878 & 0.880 & 0.894 \\
MLP & 0.841 & 0.876 & 0.860 & 0.874 & 0.863 & 0.881 & 0.924 \\
\hline
\end{tabular}

As mentioned in the feature extraction phase, there are 162 attributes obtained which from six color spaces and descriptive statistical calculation. This phase aims to find the optimal feature space from color-based features. The feature selection consists of a genetic algorithm to search for the best population. GA search for the best feature was set based on the population of each mutation. Configuration of GA was set as, maximum generation $=20$, crossover probability $=0.6$, and population size $=20$.

\subsection{Classification Modelling}

The proposed ethnicity identification used wrapper feature selection method which is embedded the classifier in the feature selection processes. For the classification modelling, we used several machine learning algorithms such as Naïve Bayes, BayesNet, k-Nearest Neighbour (k-NN), support vector machine (SVM), Random Forest and Multilayer Perceptron (MLP).

\section{RESULTS AND ANALYSIS}

The color might be affected due to several factors such as image background, eyes, mouth, lipstick, etc. In order to extract accurate skin region from the human face(s), the skin color detection was used. It was designed to detect the skin region from the image accurately by utilizing the face-skin as the initial sample for generating the skin threshold values. Thus, the skin region which segmented then exploited based on the color level. Table 2 shows the results of the identification model using skin color feature before the feature selection process during the training stage. The RGB color space achieved the highest accuracy with $84.5 \%$ using Random Forest classifier. Similarly, to $\mathrm{YCbCr}$ achieved the highest accuracy with $88.7 \%$ using Random Forest classifier. On the other hands, k-NN classifier produced the highest accuracy of $87.1 \%$ and 89.4\% under YIQ and CIE Luv color feature, respectively. Consequently, Random Forest accomplished the competitive accuracy for two color features which are HSV, and CIE Lab with respectively $88.5 \%$ and 87.8\%. Among the individual color feature, CIE Luv produced the highest accuracy when using k-NN classifier. For color-based feature, the combination color feature for trained stage achieved the highest accuracy compared to individual color features. The k-NN and Random Forest produced almost comparable accuracy with both $89.9 \%$ and $89.4 \%$. According to the results in Table 2, there are three dominant classifiers that can be used for ethnicity identification model; k-NN, Random Forest and MLP. Unfortunately, other classification algorithms such as Naïve Bayes, BayesNet and SVM have produced the lowest accuracy compared to k-NN, Random Forest and MLP. The Naïve Bayes, BayesNet and SVM classifiers only achieved $0.686,0.709$, and 0.599 respectively which are lower than 0.8 . From the results, it is shown that the color-based or chromatic-based feature has the capability to identify the ethnic group. However, color space and classification algorithm is chosen highly affects the performance of ethnicity identification.

Further investigation was done on the testing set. The testing set was prepared based on $30 \%$ of the total dataset. Table 3 shows the performance of ethnicity identification in term of accuracy of CIE Luv (best individual color space), train set, and test set in the combined color feature. Based on the results, the accuracy of the test set dropped in each classification algorithm. For instance, Naïve Bayes classifier dropped from 0.686 from to 0.574 . BayesNet classifier also dropped from 0.709 to 0.556 in the combined feature. The trends also occurred in other classifiers: SVM, k-NN, Random Forest and MLP. Among all the classifiers of the test set, Random Forest revealed the highest accuracy with 0.756. It is then followed by MLP classifier with 0.712 and $\mathrm{k}-\mathrm{NN}$ with 0.642 . However, the accuracy of ethnic identification especially in the test set is still considered low. Besides, to evaluate the performance of the proposed model, ROC analysis was done under various color space models and classification algorithms. 
Apart from the classifier perspective, Figure 2 show the ROC curves for all ethnic groups in CIE Luv color space. Visual examination on ROC curves for three classifiers implies that the Random Forest classifier has superiority over the other tested classifiers. In can be shown by the average ROC in Random Forest which achieved 0.973. The MLP classifier falls under the second with weighted ROC 0.958 and k-NN with weighted ROC 0.914. It can be concluded that Random Forest classifier and CIE Luv color space as the color feature are the best matches during the experimental. However, the ROC dropped in k-NN, Random Forest, and MLP classifier respectively at $0.650,0.853$ and 0.731 . Classifier k-NN only achieved 0.700 ROC in the test set. Meanwhile, Random Forest achieved much higher compared to k-NN with 0.881 ROC. Then followed by MLP classification with 0.855 ROC. These showed that, Random Forest classifier also performed well in a combined color feature in identifying ethnic groups.

Table 3. Comparison of the ethnicity identification in CIE Luv (TrainSet), combined color feature (TrainSet and TestSet)

\begin{tabular}{llll}
\hline Classifier & \multicolumn{3}{c}{ Color Features } \\
& CIE Luv & All (TrainSet) & All (TestSet) \\
\hline Naïve Bayes & 0.739 & 0.686 & 0.574 \\
BayesNet & 0.716 & 0.709 & 0.556 \\
SVM & 0.608 & 0.599 & 0.601 \\
$k$-NN & 0.894 & 0.899 & 0.642 \\
Random Forest & 0.880 & 0.894 & 0.756 \\
MLP & 0.881 & 0.924 & 0.712 \\
\hline
\end{tabular}

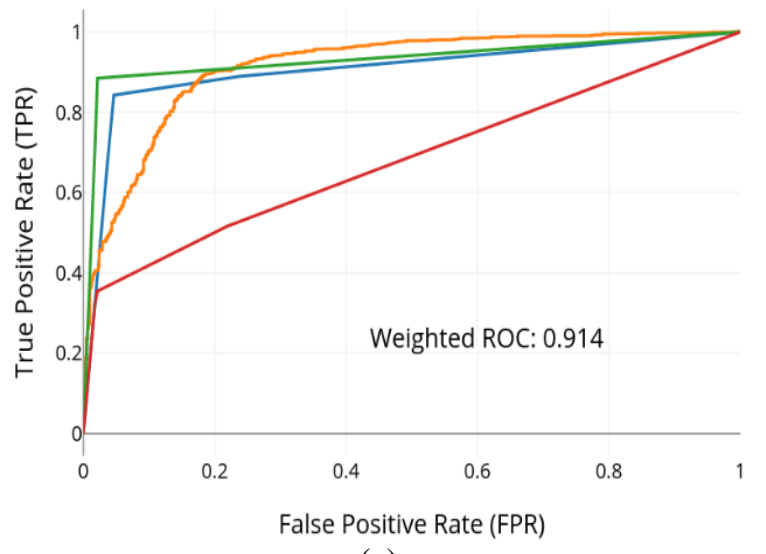

(a)

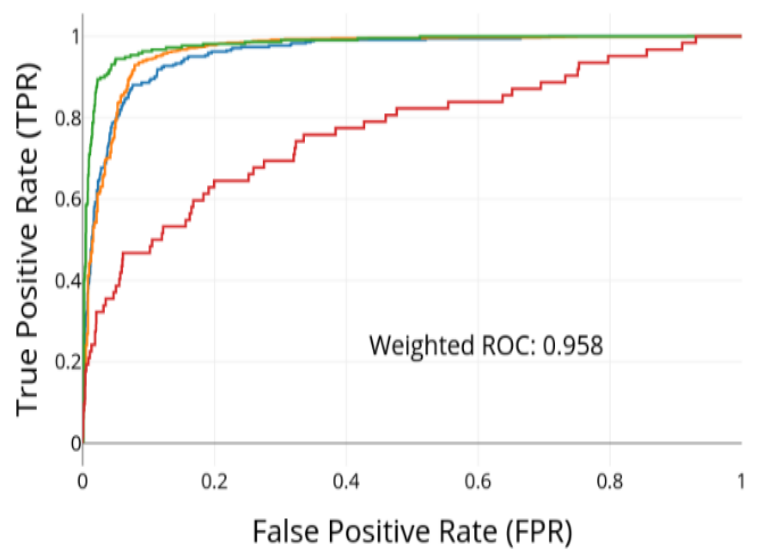

(b)

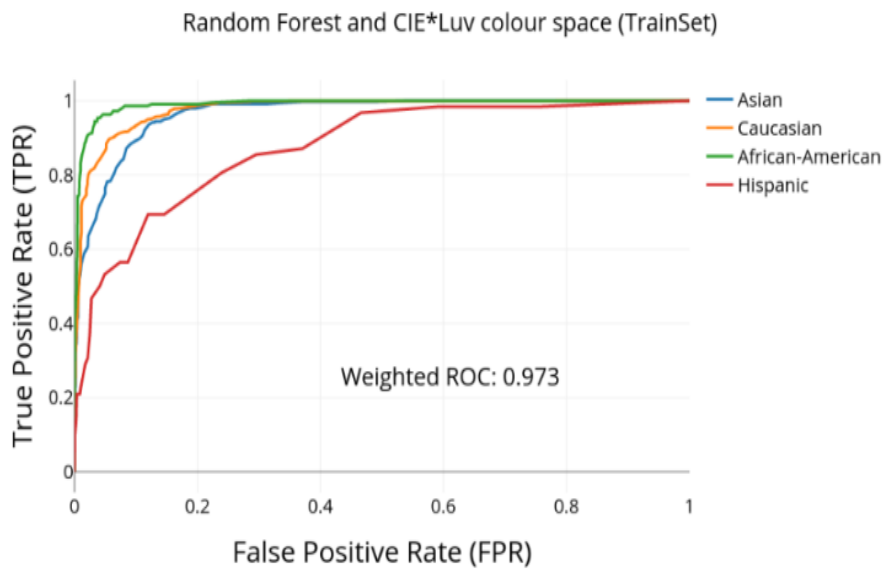

(c)

Figure 2. ROC curves of ethnicity identification for CIE Luv color space using (a) kNN (b) MLP and (c) Random Forest classifier 
To have a clearer understanding of the finding in each ethnic group, a confusion matrix was generated in testing dataset. Figure 3 shows the confusion matrix in k-NN, Random Forest and MLP classifier using the combined color feature. In Figure 3(a), the k-NN identified 67 Asian ethnics out of 147 with 0.456 TPR. Almost half of the Asian ethnic falsely identified in Caucasian (68), African (17), and Hispanic (8). For Caucasian ethnic, k-NN has successfully identified 303 out of 399 that produced 0.759 TPR. However, k-NN suffers low TPR in Hispanic ethnic that produced 1 out of 28 face images. The Hispanic ethnic was mostly classified as Caucasian ethnic. Meanwhile, the TPR result in Figure 3(b) for Asian, Caucasian, and African was reported to be better than in k-NN. Unfortunately, Random Forest suffered in identifying Hispanic ethnic which alse faced in the k-NN classifier. The Hispanic ethnic was falsely classified as Asian and Caucasian ethnic. It also occurred when using MLP classifier. The k-NN, Random Forest, and MLP classifiers suffer in characterizing the Hispanic ethnic successfully. Most of the Hispanic ethnic were falsely classified as Caucasian ethnic.

\begin{tabular}{|c|c|c|c|c|c|c|}
\hline \multirow{5}{*}{ (a) } & & Asian & Caucasian & African & Hispanic & True Positive \\
\hline & Asian & 67 & 52 & 5 & 23 & Asian: 0.456 \\
\hline & Caucasian & 68 & 303 & 0 & 28 & Caucasian: 0.759 \\
\hline & African & 17 & 16 & 55 & 2 & African: 0.611 \\
\hline & Hispanic & 8 & 19 & 0 & 1 & Hispanic: 0.036 \\
\hline \multirow{5}{*}{ (b) } & & Asian & Caucasian & African & Hispanic & True Positive \\
\hline & Asian & 90 & 52 & 5 & 23 & Asian: 0.612 \\
\hline & Caucasian & 47 & 303 & 0 & 28 & Caucasian: 0.870 \\
\hline & African & 6 & 16 & 55 & 2 & African: 0.711 \\
\hline & Hispanic & 9 & 19 & 0 & 1 & Hispanic: 0.000 \\
\hline \multirow{5}{*}{ (c) } & & Asian & Caucasian & African & Hispanic & True Positive \\
\hline & Asian & 119 & 15 & 1 & 12 & Asian: 0.810 \\
\hline & Caucasian & 47 & 290 & 6 & 56 & Caucasian: 0.727 \\
\hline & African & 18 & 8 & 62 & 2 & African: 0.689 \\
\hline & Hispanic & 12 & 14 & 0 & 2 & Hispanic: 0.071 \\
\hline
\end{tabular}

Figure 3. Confusion mattix of combined color features for (a) kNN, (b) Random Forest and (c) MLP classifier in all ethnic group

\subsection{Results on Optimal Features}

As described earlier, feature selection was implemented using wrapper subset approach. Based on the results given in Table 4, the number of selected features is significantly reduced compared to the original feature space which is 162 features in total. Naïve Bayes reduced to only nine features over 162 . The BayesNet and MLP on the other hands reduced to 8 features. Besides, Random Forest can reduce up to 12 features. Meanwhile, k-NN reduced to 20 features which is the largest selected features among the other classifiers. However, the selected features obtained were considered small compared to the original feature space.

The selected color-based feature reduced almost $93 \%$ on average of all classifiers. This mean, the majority of the color feature were irrelevant to characterise each ethnic group. For color-based feature, Random Forest showed the dominant result which produced accuracy 0.755 in the test set. The presented result in the above table indicates chromatic feature which is skin color have a good relationship with human ethnicity. Therefore the selected features are RGB (f8_G and f5_B), YCbCr (f1_Cb), YIQ (f7_Y, f2_Q, f3_Q), HSV (f7_H, f4_S), CIE Lab (f3_b) and CIE Luv (f9_L, f7_u). The component represents the color model, and the selected feature is the descriptive statistical feature corresponds to each color component. Though, it might not be significant enough to represent accurate ethnicity identification when exploiting skin color feature as the main ethnic feature vectors. Combination of color and texture feature might help to increase the performance of ethnicity identification.

Table 4. Performance Analysis of the Original Skin Color Feature Space and Optimized Feature Space Dimension

\begin{tabular}{cccc}
\hline Classifier & Original Feature Space & Reduced features & Accuracy (Train, Test) \\
\hline Naïve Bayes & & 9 & $0.686,0.574$ \\
BayesNet & \multirow{2}{*}{162} & 8 & $0.709,0.556$ \\
$k$-NN & & 20 & $0.899,0.694$ \\
Random Forest & & 11 & $0.888,0.755$ \\
MLP & 8 & $0.924,0.703$ \\
\hline
\end{tabular}




\section{CONCLUSION}

As a conclusion, type of color space model used influenced the accuracy of the model. The individual color space model does have a unique feature to characterize the ethnic group as found CIE Luv color space. Thus, color-based with combined features is much more preferred compared to the individual color model. The initial findings in the proposed ethnicity identification using color-based as the key feature have shown a limitation in producing a reasonable performance in the accuracy perspective. As seen in the results, the accuracy and the ROC decreased significantly because the identification of ethnicity failed to classify, especially in the successful Hispanic group. For this reason, we would like to improve the identification performance of the ethnic group by examining the additional features available especially from the face texture features.

\section{ACKNOWLEDGEMENTS}

We would like to show our gratitude to Universiti Malaysia Pahang (RDU vote number RDU1803142) for supporting this research.

\section{REFERENCES}

[1] Bekhouche, S.E., et al., "Pyramid multi-level features for facial demographic estimation". Expert Systems with Applications, 2017; 80: 297-310.

[2] Antipov, G., et al., "Effective training of convolutional neural networks for face-based gender and age prediction". Pattern Recognition, 2017; 72: 15-26.

[3] Mohamad, F.S., M. Iqtait, and F. Alsuhimat. "Age prediction on face features via multiple classifiers". 2018. IEEE.

[4] Hajibabaei, M., A. Volokitin, and R. Timofte. "Early adaptation of deep priors in age prediction from face images". 2017.

[5] Pantraki, E. and C. Kotropoulos. "Multi-way regression for age prediction exploiting speech and face image information". IEEE. 2017.

[6] Trivedi, A. and D.G. Bessie Amali. "A comparative study of machine learning models for ethnicity classification". 2017.

[7] Chen, H., et al., "A Novel Race Classification Method Based on Periocular Features Fusion". International Journal of Pattern Recognition and Artificial Intelligence, 2017; 31(08): 1750026.

[8] Tariq, U., Y. Hu, and T.S. Huang. "Gender and ethnicity identification from silhouetted face profiles". in Image Processing (ICIP), 2009 16th IEEE International Conference on. 2009. IEEE.

[9] Guo, G. and G. Mu. "A study of large-scale ethnicity estimation with gender and age variations". in Computer Vision and Pattern Recognition Workshops (CVPRW), 2010 IEEE Computer Society Conference on. 2010. IEEE.

[10] Boyseens, A. and S. Viriri. "Component-Based Ethnicity Identification from Facial Images". in International Conference on Computer Vision and Graphics. 2016. Springer.

[11] Masood, S., et al., Prediction of Human Ethnicity from Facial Images Using Neural Networks, in Data Engineering and Intelligent Computing. 2018, Springer. pp. 217-226.

[12] Carcagnì, P., et al., "A study on different experimental configurations for age, race, and gender estimation problems". EURASIP Journal on Image and Video Processing, 2015; 2015(1): 37.

[13] Anwar, I. and N.U. Islam, "Learned features are better for ethnicity classification". Cybernetics and Information Technologies, 2017; 17(3): 152-164.

[14] $\mathrm{Fu}, \mathrm{S}$., H. He, and Z.-G. Hou, "Learning race from face: A survey". IEEE transactions on pattern analysis and machine intelligence, 2014; 36(12): 2483-2509.

[15] Ernawan, F., "Tchebichef image watermarking along the edge using YCoCg-R color space for copyright protection". International Journal of Electrical and Computer Engineering (IJECE), 2019; 9(3): 1850.

[16] Hdioud, B., et al., "Detecting and Shadows in the HSV Color Space Using Dynamic Thresholds". Bulletin of Electrical Engineering and Informatics (BEEI), 2018; 7(1): 70-79.

[17] Dewi, A.F., F. Arnia, and R. Muharar, "Effectiveness of MPEG-7 Color Features in Clothing Retrieval". Bulletin of Electrical Engineering and Informatics (BEEI), 2017; 6(2): 166-173.

[18] Farag, M.S., M.M. El Din, and H. El Shenbary, "Parking entrance control using license plate detection and recognition". Indonesian Journal of Electrical Engineering and Computer Science (IJEECS), 2019; 15(1): 476-483.

[19] Hosoi, S., E. Takikawa, and M. Kawade. "Ethnicity estimation with facial images". in Automatic Face and Gesture Recognition, 2004. Proceedings. Sixth IEEE International Conference on. 2004. IEEE.

[20] Stavrianos, C., et al., "Facial Anatomy and Mapping Across Races' C. Stavrianos," C. Papadopoulos," O. Pantelidou,"I. Emmanouil and "N. Petalotis" Department of Endodology (Forensic Odontology), "Department of Removable Prosthodontics". 2012.

[21] Xiao, K., et al., "Characterising the variations in ethnic skin colours: a new calibrated data base for human skin". Skin Research and Technology, 2017; 23(1): 21-29.

[22] Hill, H., V. Bruce, and S. Akamatsu, "Perceiving the sex and race of faces: The role of shape and colour". Proceedings of the Royal Society of London B: Biological Sciences, 1995; 261(1362): 367-373.

[23] Roomi, S.M.M., et al. "Race classification based on facial features". in Computer Vision, Pattern Recognition, Image Processing and Graphics (NCVPRIPG), 2011 Third National Conference on. 2011. IEEE. 
[24] Malskies, C.R., E. Eibenberger, and E. Angelopoulou, "The recognition of ethnic groups based on histological skin properties". 2011.

[25] Xie, Y., K. Luu, and M. Savvides. "A robust approach to facial ethnicity classification on large scale face databases". in Biometrics: Theory, Applications and Systems (BTAS), 2012 IEEE Fifth International Conference on. 2012. IEEE.

[26] Anzures, G., et al., "Minimizing Skin Color Differences Does Not Eliminate the Own-Race Recognition Advantage in Infants". Infancy, 2011; 16(6): 640-654.

[27] Brooks, K.R. and O.S. Gwinn, "No role for lightness in the perception of black and white? Simultaneous contrast affects perceived skin tone, but not perceived race". Perception, 2010; 39(8): 1142-1145.

[28] Osman, M.Z., M.A. Maarof, and M.F. Rohani, "Improved Dynamic Threshold Method for Skin Colour Detection Using Multi-Colour Space". American Journal of Applied Sciences, 2016; 13(2): 135-144.

[29] Osman, M.Z., M.A. Maarof, and M.F. Rohani. "Improved Skin Detection based on Dynamic Threshold using MultiColour Space". in 2014 International Symposium on Biometrics and Security Technologies. 2014.

[30] Osman, M.Z., et al., "Multi-Scale Skin Sample Approach for Dynamic Skin Color Detection: An Analysis". Advanced Science Latters, 2018; 24(10): 7662-7667(6).

\section{BIOGRAPHIES OF AUTHORS}
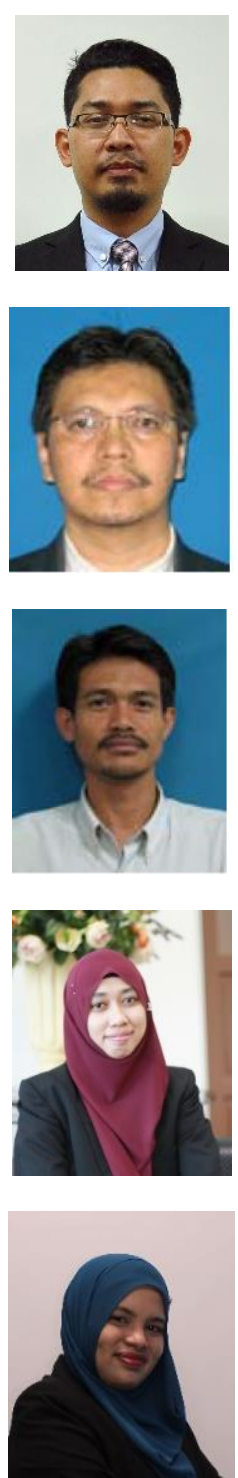

Mohd Zamri Osman received his Ph.D degree from Universiti Teknologi Malaysia in 2017. He also obtained Master of Computer Science (Information Security) from the same university. Currently, he worked as senior lecturer at Faculty of Computer Systems and Software Engineering at Universiti Malaysia Pahang, Malaysia. His research interest is on image processing, machine learning, pattern recognition, and information security.

Mohd Aizaini Maarof is a Professor at School of Computing, Universiti Teknologi Malaysia (UTM). He obtained his B.Sc (Computer Science) and M.Sc (Computer Science) from U.S.A and his Ph.D degrees from Aston University, Birmingham, United Kingdom in the area of Information Technology (IT) Security. Currently, his research involves in the areas of Network Security, Web Content Filtering, MANET Security and Cryptography.

Mohd Fo'ad Rohani obtained his B.E (Electrical) from University Malaya (UM) and M.Sc (Electronic) from University of Wales, Cardiff, UK and Ph.D (Comp Sc.) degree from Universiti Teknologi Malaysia (UTM). Currently, he is a Senior Lecturer and holding position as Associate Chair (Facility) at the School of Computing, Faculty of Engineering, UTM. He is also a research member of Information Assurance and Security Research Group, UTM. His research interests include Network Security, Pattern Recognition, and Internet of Things.

Nilam Nur Amir Sjarif is a senior lecturer at Razak Faculty of Technology and Informatics, under the department of Advanced Infomatics, Universiti Teknologi Malaysia, Kuala Lumpur. She received her bachelor and PhD from the Universiti Teknologi Malaysia in 2010 and 2015, respectively. She is active in the research field Image and Video Processing, Pattern Recognition, Machine Learning, Data Mining and Business Intelligence.

Nor Saradatul Akmar distinctively received her PhD from University Teknologi Malaysia (UTM), Malaysia. She also obtained her First Degree of Electronic Engineering (Electric-Medical Elctronic) from the same university. She is currently a senior lecturer at the Faculty of Computer Systems and Software Engineering at Universiti Malaysia Pahang, Malaysia. Her area of research includes Image Processing (mostly in medical and neuro-images) and Wireless Sensor Network. 